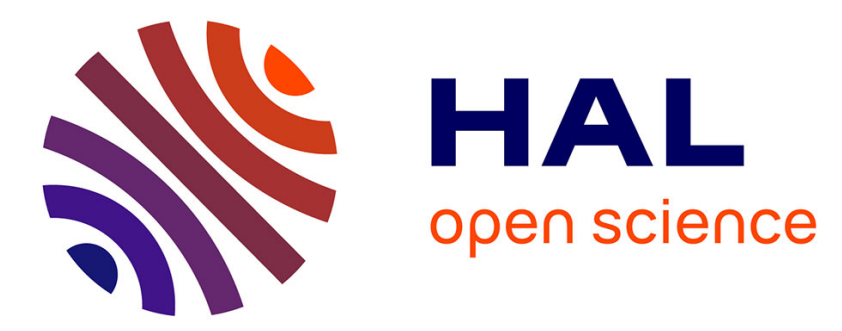

\title{
Helical-Ring Antenna for Hemispherical Radiation in Circular Polarization
}

Christophe Morlaas, Bernard Souny, Alexandre Chabory

\section{To cite this version:}

Christophe Morlaas, Bernard Souny, Alexandre Chabory. Helical-Ring Antenna for Hemispherical Radiation in Circular Polarization. IEEE Transactions on Antennas and Propagation, 2015, PP (99), pp.1. 10.1109/TAP.2015.2479640 . hal-01207649

\section{HAL Id: hal-01207649 \\ https://hal-enac.archives-ouvertes.fr/hal-01207649}

Submitted on 19 Oct 2015

HAL is a multi-disciplinary open access archive for the deposit and dissemination of scientific research documents, whether they are published or not. The documents may come from teaching and research institutions in France or abroad, or from public or private research centers.
L'archive ouverte pluridisciplinaire HAL, est destinée au dépôt et à la diffusion de documents scientifiques de niveau recherche, publiés ou non, émanant des établissements d'enseignement et de recherche français ou étrangers, des laboratoires publics ou privés. 


\title{
Helical-Ring Antenna for Hemispherical Radiation in Circular Polarization
}

\author{
Christophe Morlaas, Bernard Souny, and Alexandre Chabory
}

\begin{abstract}
A compact helical-ring antenna is proposed to realize a Huygens source in circular polarization. The radiation pattern of this antenna is mainly directed toward an half-space without the use of any reflector. The principle relies on the combination of two sets of collocated electric and magnetic short dipoles. The helical ring is excited by means of in-quadrature capacitive probes. Solutions are proposed to adjust the resonant frequency and balance the contributions of the magnetic and electric dipoles. The direction of the radiation pattern can be electrically switched frontward or backward by modifying the in-quadrature probes. Besides, the size of the antenna can be reduced by increasing the number of turns, therefore without high-permittivity materials. The properties of this antenna are analyzed by means of simulations and confirmed via measurements on a prototype.
\end{abstract}

Index Terms-Antenna radiation patterns, Helical antennas, Dipole antennas, Huygens source, Circular polarization.

\section{INTRODUCTION}

$\mathbf{F}$ OR some applications, antennas must be as compact as possible with a radiation in circular polarization mainly directed toward an half-space. Such criteria exist for RFID [1], [2], GNSS or satellite-aided search and rescue systems. They are also important for mobile handsets or body network antennas that must respect SAR constraints [3].

Few designs achieve to meet all these criteria. For instance, a well-known technique to obtain an half-space radiation in circular polarization is to add a reflector or a ground plane. However this significantly increases the total size of the antenna. Another technique consists in using a quadrifilar helix antenna [4], [5]. Their length however limits their possible integration in mobile systems.

In 2010, two publications have independently presented antennas radiating circular polarization in an half-space. Alitalo et $a l$. have proposed an antenna based on two orthogonal wires and two orthogonal loops (chiral particle) to obtain a Huygens source [6]. A detailed study of their solution is presented in [7]. Theoretically, this design gives a very good axial ratio but the realized prototype suffers from a lack of symmetry due to the finite size of the elements. As a consequence, the polarization is not strictly circular in any direction of space. In the same year and independently, we have proposed another solution consisting in an helical ring to achieve a half-space radiation in circular polarization [8], [9]. The design of this

C. Morlaas and A. Chabory are with ENAC, TELECOM-EMA, Toulouse, F-31055 France e-mail: morlaas@recherche.enac.fr. They are also affiliated with Université de Toulouse, F-31400 Toulouse, France.

B. Souny is an associate researcher with ENAC, TELECOM-EMA. antenna presents a natural symmetry. This work was inspired by the articles of Wheeler and Krauss [10], [11], where a helix of small size has been used to realize collocated electric and magnetic short dipoles.

In this article, from theoretical derivations, we explain why helical ring antennas can radiate as a Huygens source in circular polarization. We verify this statement by means of simulations firstly on a simple helical-ring wire and then on more realistic antennas including feeding probes. We show that the direction of the radiation pattern can be electrically switched frontward or backward. Besides, the sense of the circular polarization is determined by the orientation of the helix. We explain how to adjust the resonant frequency and balance the contributions of the electric and magnetic dipoles. Measurements on a prototype are also presented to confirm simulation results.

In Section II, we explain how short dipoles can be arranged so as to obtain an hemispherical radiation in circular polarization. In Section III, helical rings are presented. In Section IV, we confirm via simulations the radiation characteristics of an ideal and isolated helical-ring wire. In Section V, we compare measurements and simulations for a 4-turns helical ring fed by two capacitive probes. In Section VI, the influence of the number of turns is studied.

\section{HEMISPHERICAL RADIATION IN CIRCULAR POLARIZATION WITH COLLOCATED SHORT DIPOLES}

\section{A. Circular polarization}

One way to obtain a radiation in circular polarization is to collocate one electric and one magnetic short dipoles. In the far-field, the electric field of such dipoles at a point $r$ is given by

$$
\boldsymbol{E}_{\mathrm{d} z}(\boldsymbol{r})=-k^{2} c \frac{e^{-j k r}}{4 \pi r}\left[\hat{\boldsymbol{r}} \times \boldsymbol{p}_{\mathrm{m}}+\zeta \hat{\boldsymbol{r}} \times\left(\hat{\boldsymbol{r}} \times \boldsymbol{p}_{\mathrm{e}}\right)\right],
$$

where $k$ is the wavenumber, $c$ is the speed of light, $\zeta$ is the wave impedance, $r=\|\boldsymbol{r}\|$, and $\hat{\boldsymbol{r}}=\boldsymbol{r} / r$. Furthermore, $\boldsymbol{p}_{\mathrm{e}}$ and $p_{\mathrm{m}}$ represent the moments of the electric and magnetic dipoles, respectively. Upon assuming dipoles oriented along the $z$-axis, i.e. $\boldsymbol{p}_{\mathrm{e}}=p_{\mathrm{e}} \hat{\boldsymbol{z}}$ and $\boldsymbol{p}_{\mathrm{m}}=p_{\mathrm{m}} \hat{\boldsymbol{z}}$, the electric field can be written in spherical coordinates as

$$
\boldsymbol{E}_{\mathrm{d} z}=A \sin \theta \frac{e^{-j k r}}{4 \pi r}\left(\hat{\boldsymbol{\theta}}-\alpha_{\mathrm{p}} \hat{\boldsymbol{\phi}}\right)
$$

with $A=-k^{2} c \zeta p_{\mathrm{e}},(\hat{\boldsymbol{\theta}}, \hat{\boldsymbol{\phi}})$ the unit vectors of spherical coordinates, and

$$
\alpha_{\mathrm{p}}=\frac{p_{\mathrm{m}}}{\zeta p_{\mathrm{e}}} .
$$


From (2), the polarization remains the same in all directions of space, and is simply controlled by the value of $\alpha_{\mathrm{p}}$. Circular polarization is obtained for $\alpha_{\mathrm{p}}= \pm j$ fixed.

\section{B. Half-space radiation}

The objective of this article is to obtain a circularlypolarized antenna with an half-space radiation, i.e. which radiation is quasi-omnidirectional in an half-space, and weak in the other half-space. This can be achieved by combining twice the previous set of short dipoles. The first set corresponds to electric and magnetic dipoles oriented along the $x$-axis. The second one corresponds to identical dipoles oriented along the $y$-axis and fed in phase quadrature. Before studying the general case, the principle is firstly presented for $\alpha_{\mathrm{p}}=j$. As illustrated in Fig. 1, in this case, from (1), the electric field of the $x$-directed dipoles is proportional to $-\hat{\boldsymbol{x}} \pm j \hat{\boldsymbol{y}}$ along the $\pm z$ axis, respectively. For a quadrature feeding of $-j$, the electric field of the $y$-directed dipoles is proportional to $\mp \hat{\boldsymbol{x}}+j \hat{\boldsymbol{y}}$. Finally, along $+z$ the contributions of $x$-directed and $y$-directed dipoles add together while along $-z$ these contributions cancel each other out, which is consistent with the expected half-space radiation.

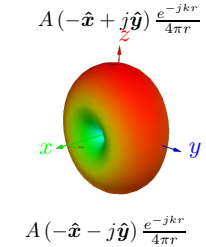

(a) $x$-directed dipoles

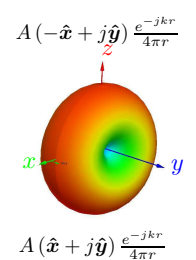

(b) $y$-directed dipoles

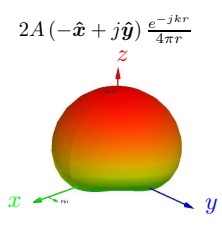

Fig. 1. Hemispherical radiation pattern: contribution of $x$-directed and inquadrature $y$-directed dipoles in the electric field.

In the general case, the directions of the maximum-zero depend on the feeding and polarization. To demonstrate this property, the electric field of the first set of dipoles is developed in spherical coordinates. Considering $x$-directed dipoles in (1), because $\alpha_{\mathrm{p}}= \pm j$, this yields

$$
\boldsymbol{E}_{\mathrm{d} x}=A \frac{e^{-j k r}}{4 \pi r}\left(-\cos \theta \cos \phi+\alpha_{\mathrm{p}} \sin \phi\right)\left(\hat{\boldsymbol{\theta}}-\alpha_{\mathrm{p}} \hat{\boldsymbol{\phi}}\right) .
$$

Similarly, the electric field of the second set of dipoles can be written as

$$
\boldsymbol{E}_{\mathrm{d} y}=A \frac{e^{-j k r}}{4 \pi r}\left(-\cos \theta \sin \phi-\alpha_{\mathrm{p}} \cos \phi\right)\left(\hat{\boldsymbol{\theta}}-\alpha_{\mathrm{p}} \hat{\boldsymbol{\phi}}\right) .
$$

Adding both contributions yields

$$
\boldsymbol{E}=\boldsymbol{E}_{\mathrm{d} x}+\alpha_{\mathrm{f}} \boldsymbol{E}_{\mathrm{d} y},
$$

where $\alpha_{\mathrm{f}}= \pm j$ characterizes the in-quadrature relative feedings. Inserting (4) and (5) in (6), the total electric field can be written as

$$
\boldsymbol{E}=-A \alpha_{\mathrm{f}} \alpha_{\mathrm{p}} e^{\alpha_{\mathrm{f}} \phi}\left(1+\alpha_{\mathrm{f}} \alpha_{\mathrm{p}} \cos \theta\right)\left(\hat{\theta}-\alpha_{\mathrm{p}} \hat{\phi}\right) \frac{e^{-j k r}}{4 \pi r} .
$$

Finally, as in (2), the polarization remains the same regardless of the direction of space and is circular. For in-quadrature feedings and circular polarization, we have $\alpha_{\mathrm{p}} \alpha_{\mathrm{f}}= \pm 1$. Therefore, the magnitude of the electric field is given by

$$
\|\boldsymbol{E}\|=\frac{|A|}{4 \pi r} \begin{cases}1+\cos \theta & \text { for } \alpha_{\mathrm{f}} \alpha_{\mathrm{p}}=1 \\ 1-\cos \theta & \text { for } \alpha_{\mathrm{f}} \alpha_{\mathrm{p}}=-1\end{cases}
$$

This means that the antenna constitutes a Huygens source, i.e. its pattern is simply given by a cardioid. Besides the pattern orientation depends on the value of $\alpha_{\mathrm{f}} \alpha_{\mathrm{p}}$ :

- For $\alpha_{\mathrm{f}} \alpha_{\mathrm{p}}=+1$, the field is mainly directed toward the $z>0$ half-space. The radiation is null for $z \rightarrow-\infty$ and maximal for $z \rightarrow+\infty$;

- For $\alpha_{\mathrm{f}} \alpha_{\mathrm{p}}=-1$, the field is mainly directed toward the $z<0$ half-space. The radiation is null for $z \rightarrow+\infty$ and maximal for $z \rightarrow-\infty$.

Hence the direction of the radiation pattern can easily be electrically switched by modifying the value of $\alpha_{\mathrm{f}}$. In Table I, the radiation properties of the antenna are listed according to the feeding and polarization. To confirm that this antenna mainly radiates toward one half-space, we express the ratio between the power directed toward the maximal radiation halfspace $P_{1 / 2}$ and the total radiated power $P_{\mathrm{t}}$. Integrating the cardioid pattern over a sphere, we obtain

$$
\frac{P_{1 / 2}}{P_{\mathrm{t}}}=\frac{\int_{0}^{\pi / 2}(1+\cos \theta)^{2} \sin \theta d \theta}{\int_{0}^{\pi}(1+\cos \theta)^{2} \sin \theta d \theta}=\frac{7}{8}=87.5 \% .
$$

Thus, $87.5 \%$ of the power is radiated toward one half-space. From (8), the maximal directivity is given by

$$
D_{\max }=\frac{4 \pi U_{\max }}{P_{\mathrm{t}}}=\frac{8}{\int_{0}^{\pi}(1+\cos \theta)^{2} \sin \theta d \theta}=3,
$$

where $U_{\max }=\max \|E\|^{2} /(2 \zeta)$ is the maximal radiation intensity. In $\mathrm{dBi}$, this corresponds to $D_{\max \mathrm{dBi}}=4.77 \mathrm{dBi}$.

\begin{tabular}{c|c|c} 
& $\alpha_{\mathrm{f}}=-j$ & $\alpha_{\mathrm{f}}=+j$ \\
\hline$\alpha_{\mathrm{p}}=+j$ & RHCP - upward & RHCP - downward \\
$\alpha_{\mathrm{p}}=-j$ & LHCP - downward & LHCP - upward \\
& TABLE I &
\end{tabular}

RADIATION PROPERTIES ACCORDING TO THE TYPE OF POLARIZATION.

\section{HELICAL RING ANTENNA}

\section{A. Helical antenna}

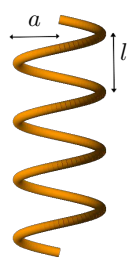

(a) helix

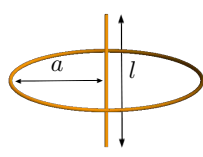

(b) equivalent model for one turn
Fig. 2. Helical antenna and its equivalent model.

One way to realize a set of two collocated and aligned short dipoles is to use a helical antenna as shown by Wheeler [10] 
and Kraus [11]. Indeed, as illustrated in Fig 2, a helix can be considered as a series of loops and straight wires upon assuming the length of one turn is small compared to the wavelength so that the current is uniform along the turn. Each turn of the helix is then equivalent to a straight wire and a loop, which correspond to an electric and a magnetic short dipoles, respectively. The moment of the electric dipole is related to the helix properties by

$$
p_{\mathrm{e}}=\frac{I l}{j \omega},
$$

where $I$ is the current circulating along the turn and $l$ the helix pitch, i.e. the length of one turn measured parallel to the helix axis. The moment of the magnetic dipole is given by

$$
p_{\mathrm{m}}=\left\{\begin{aligned}
-\zeta I S / c & \text { for a left-hand helix, } \\
\zeta I S / c & \text { for a right-hand helix. }
\end{aligned}\right.
$$

where $S$ is the area of the loop. From the expressions of the moments (11) and (12), the dipoles present a natural phase quadrature and the condition (3) to have a circular polarization reduces to

$$
S=\frac{\lambda l}{2 \pi},
$$

where $\lambda$ is the wavelength. This means that circular polarization can be obtained by choosing the loop area $S$ and pitch $l$ accordingly. Then, the sense of the circular polarization only depends on the type of helix:

- left-hand circular polarization for a left-hand helix;

- right-hand circular polarization for a right-hand helix. The value of $\alpha_{\mathrm{p}}$ is then given by

$$
\alpha_{\mathrm{p}}= \begin{cases}+j & \text { right-hand helix and polarization } \\ -j & \text { left-hand helix and polarization. }\end{cases}
$$

\section{B. Resonant helical ring}

A helix with a short turn length can be used to realize a set of two collocated and aligned short dipoles. Thus, the principle exposed in Section II-B, can be realized by means of collocated and orthogonal helices. Besides, for the combination of helices to be used as a matched antenna, it is excited near self resonance. Finally, the antenna must be a resonant structure of helical shape with two orthogonal modes. In order to prevent unwanted mode coupling, the combination of helices must respect a rotational symmetry of $90^{\circ}$. As shown in Fig. 3a, the simplest solution consists in a crossshaped structure. However, that requires a crossing between both helices at the antenna center, which renders difficult to maintain the symmetry in any practical design. Alternatively, as exposed in Fig. 3b and Fig. 3c, a square or a circular ring can be used. In this article, the circular ring is preferred because it yields a more-symmetric and simpler design.

The shape of the turns is chosen as elliptic. The ellipse flatness will be used as an additional parameter to balance the contributions of the electric and magnetic dipoles. Finally, the antenna is described by a wire of equation

$$
\begin{aligned}
& x=[d+a \cos (N \xi)] \cos \xi, \\
& y=[d+a \cos (N \xi)] \sin \xi, \quad \text { for } \xi \in[0,2 \pi[, \\
& z= \pm b \sin (N \xi),
\end{aligned}
$$

where $N$ is the number of turns, $d$ the ring mean radius, and $a, b$ the semi-axes of the elliptic turns. In this equation the sign of the $z$-component corresponds to either a left or a righthanded helix. To obtain a rotational symmetry of $90^{\circ}, N$ must be a multiple of 4 .

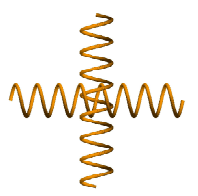

(a) cross

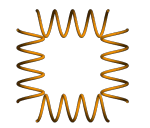

(b) square

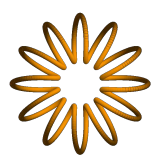

(c) ring
Fig. 3. Different helix arrangements.

\section{Conditions to obtain circular polarization at self resonance}

In this section, conditions to obtain self-resonance and circular polarization at a given frequency are derived. They are used to obtain approximate values of the parameters $a, b$ and $d$. When a straight helix is fed at its center, self-resonance roughly occurs when the total length of the wire is half a wavelength [10]. By analogy, the helical-ring resonance should correspond to a total length of one wavelength because the resonance of a ring is approximately reached for a circumference of one wavelength [12]. This condition can be written as

$$
N C=\lambda,
$$

where $C$ is the wire length for one turn. Besides, since the helix pitch is of $l=2 \pi d / N$, the condition to have circular polarization (13) reduces to

$$
S=\frac{\lambda d}{N} .
$$

To have circular polarization at self-resonance, (16) and (17) must be satisfied simultaneously. When the turns are of circular shape $(a=b), C$ and $S$ can be approximated by the perimeter and area of a circle, i.e. $S=\pi a^{2}$ and $C=2 \pi a$. Using (16) and (17), this yields

$$
a=b=\frac{\lambda}{2 \pi N}, \quad d=\frac{\lambda}{4 \pi N} .
$$

This renders difficult any practical design because the helix radius is twice as large as the ring radius, which means that the turns overlap. To maintain circular polarization at selfresonance with non-overlapping turns, a solution is to increase $b$ with respect to $a(b>a)$. To do so, we fix $a=\eta d$ with $\eta<1$. If we assume that $S \approx \pi a b$ and $C \approx 2 \pi \sqrt{a b}$, we obtain

$$
a=\eta \frac{\lambda}{4 \pi N}, \quad b=\frac{\lambda}{\eta \pi N}, \quad d=\frac{\lambda}{4 \pi N} .
$$

\section{Choice of the antenna parameters}

From the results of the previous section, we here explain how to set the antenna characteristics at a given frequency $f_{0}$.

Firstly, the choice of the turn number $N$ results from a tradeoff between size and bandwidth: when $N$ is increased, the antenna size decreases (19) at the cost of a reduced bandwidth. As previously explained, the parameter $\eta$ should be near 
one. Nevertheless, technical constraints when realizing nearly overlapping turns must also be accounted. Note that when $\eta \rightarrow 1$, (16) may lose accuracy because inter-loop couplings will significantly influence the resonance frequency.

When $N$ and $\eta$ are fixed, the expressions (19) can be used to estimate approximate values for $a, b$ and $d$. These values constitute a first guess that must be improved via simulations. To obtain the circular polarization, the parameters must be slightly modified so as to balance the contributions of the electric and magnetic moments at self-resonance. From (11) and (12), the influence of the sizes on these moments are as follows:

- An increase of $d$ yields a larger helix pitch $l$. This results in a stronger electric moment.

- An increase of $a$ or $b$ yields a larger loop area $S$. This results in a stronger magnetic moment.

Note that a side effect counteracts the stronger magnetic moment when $a$ or $b$ are increased. Indeed, since the antenna wire becomes longer, the resonance frequency decreases, which results in a stronger electric moment (12). Because $a$ is fixed with respect to $d$ by $\eta$, the simplest way to tune the polarization is to play with $b$. When the polarization becomes circular, a scaling factor on $a, b$ and $d$ is finally applied to reset the resonance frequency at the expected value.

\section{PROOF OF CONCEPT}

\section{A. Configuration}

To confirm the theoretical characteristics presented in the previous section, an antenna constituted by a wire of equation (15) is considered (Fig. 4). The feeding is modeled by 4 voltage sources $V_{0}, V_{1}, V_{2}$ and $V_{3}$ placed along the wire at $\xi=0, \pi / 2, \pi$ and $3 \pi / 2$, respectively. The simulations are realized by means of the method of moments with Feko. The number of turns is $N=4$. The helix is right-handed so that $\alpha_{\mathrm{p}}=j$.

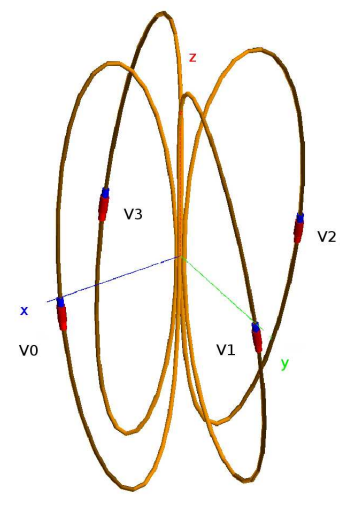

Fig. 4. Four-turns helical ring antenna.

We want to reach a resonance at a frequency of $0.59 \mathrm{GHz}$. We impose $\eta=0.95$. Using (19), we find $a=9.61 \mathrm{~mm}, b=$ $42.59 \mathrm{~mm}$ and $d=10.12 \mathrm{~mm}$. After optimization via simulations, the antenna dimensions are of $a=7.8 \mathrm{~mm}$, $b=27.28 \mathrm{~mm}$ and $d=8.2 \mathrm{~mm}$. Thus, the analytical expressions overestimate the optimized antenna dimensions by factors of about $20 \%-35 \%$. This can be explained by approximations in the calculation. Inter-loop couplings are notably neglected even though they significantly affect the resonance frequency in this case because $\eta$ is almost equal to one.

\section{B. Simulation results}

The analysis is firstly led when only the mode associated with $x$-directed dipoles is excited. This is achieved for $V_{2}=-V_{0}$ and $V_{1}=V_{3}=0$. In Fig. 5, the radiation pattern is observed in the $x 0 z$ plane. The result is consistent with theory, i.e. the polarization is circular for any direction of space, and the field is null along the dipole axis. Even if the turn length $C \approx 2 \pi \sqrt{a b} \approx 0.18 \lambda$ is not strictly negligible, the simulation confirms this length is sufficiently small for the helical ring to radiate as short dipoles.

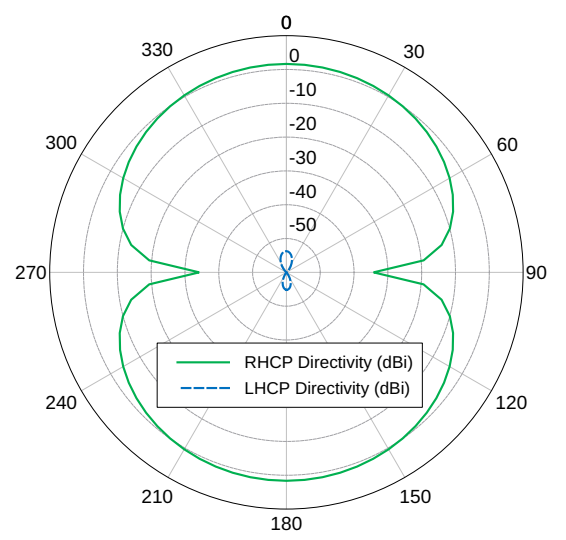

Fig. 5. Directivity pattern $(\mathrm{dBi})$ of one mode in the $x O z$ plane at $0.59 \mathrm{GHz}$.

Secondly, we consider the case where both modes are excited in phase quadrature. For $\alpha_{\mathrm{f}}= \pm j$, this corresponds to $V_{n}=V_{0} \exp ( \pm j(n-1) \pi / 2)$, with $n=0, \cdots, 3$. In Fig. 6 , the radiation pattern associated with $\alpha_{\mathrm{f}}=-j$ and $+j$ are displayed in the $x O z$ plane. The field magnitude is consistent with the cardioid pattern (8). For both feedings, the maximal directivity obtained via simulations is about $4.7 \mathrm{dBi}$ which is in agreement with the theoretical result (10). Besides, the radiation is mainly oriented toward one half-space and the direction of the radiation pattern can be electrically switched frontward or backward by modifying the feeding.

\section{Modified design}

In the previous design, to obtain circular polarization, the dimensions have been chosen so that the magnetic moments are strong enough to balance with the electric moments. This has yielded a large helix cross section with a strong ellipticity. In this case, the inner radius of the ring $d-a=0.4 \mathrm{~mm}$ is small. Thus, the self-resonance frequency strongly depends on 


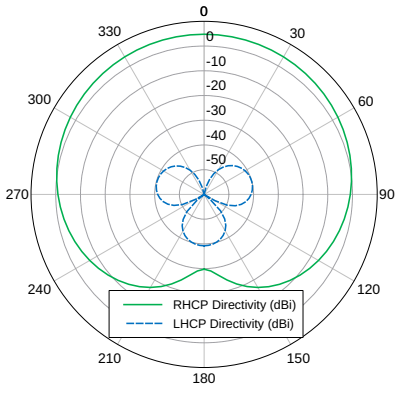

(a) $\alpha_{\mathrm{f}}=-j$

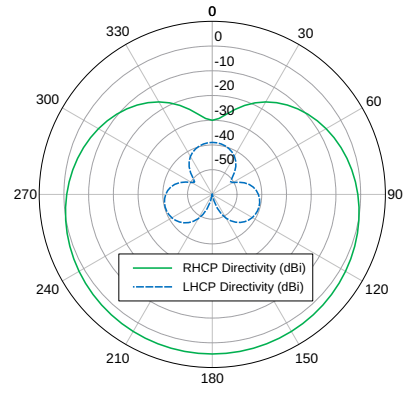

(b) $\alpha_{\mathrm{f}}=+j$ (b) Directivity pattern $(\mathrm{dBi})$ in the $x O z$ plane for $\alpha_{\mathrm{f}}=-j$ at $0.59 \mathrm{GHz}$.

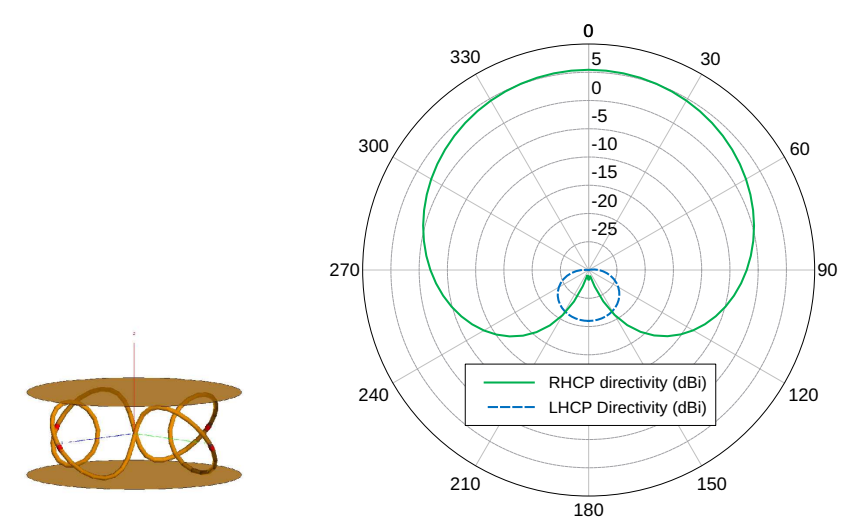

the inter-wires capacitors occurring at the center of the ring. the axial ratio, two circular metallic disks can be added above and below the ring to capacitively load the ring. Their radii are chosen close to the ring outer radius $d+a$. The gap between the ring and the disks is chosen small with respect to the wavelength. Due to the image principle, the disks strengthen the magnetic moments and weaken the electric moments. Thus, circular polarization is obtained for a smaller helix cross section, which means that both $a$ and $b$ can be reduced with respect to $d$. This decreases the inter-turns couplings at the center of the ring.

To choose the parameters, the expressions (19) can still be used as a first guess even if they do not take into account the disks. The parameter values must then be optimized by means of simulations using the rules described in Section III-D.

In Fig. 7a, a helical ring with two disks is considered at $0.59 \mathrm{GHz}$. The dimensions are $a=16.3 \mathrm{~mm}, b=22.78 \mathrm{~mm}$ and $d=27.15 \mathrm{~mm}$. Besides, the disks radii are $a+d$ and the gaps between the ring and the disks are of $3.318 \mathrm{~mm}$. The radiation pattern for $\alpha_{\mathrm{f}}=-j$ in the $x O z$ plane is plotted in Fig. 7b. The radiation pattern is consistent with the theoretical result (8). Hence, the antenna principle remains valid in the presence of the disks. The radiated field remains mainly RHCP and directed only toward the $z>0$ half-space. The maximal directivity obtained via simulations is about $5.4 \mathrm{dBi}$.

\section{REALISTIC HELICAL RING ANTENNA WITH 4-TURNS}

The aim of this section is to present the design, simulation, realization and measurement of a 4-turns helical ring antenna. For the simplicity of realization, only one metallic disk and only two feed probes (one per mode) are used to excite the antenna.

\section{A. Configuration}

In Fig. 8, we display the 3D model and a picture of the prototype. More details of the structure are presented in Fig. 9 where a side and two top views are displayed. From bottom to top, the antenna is constituted by the 4 following layers:

- layer 1: an aluminum square support of side length $48 \mathrm{~mm}$ and thickness $5 \mathrm{~mm}$; (a) 3D view

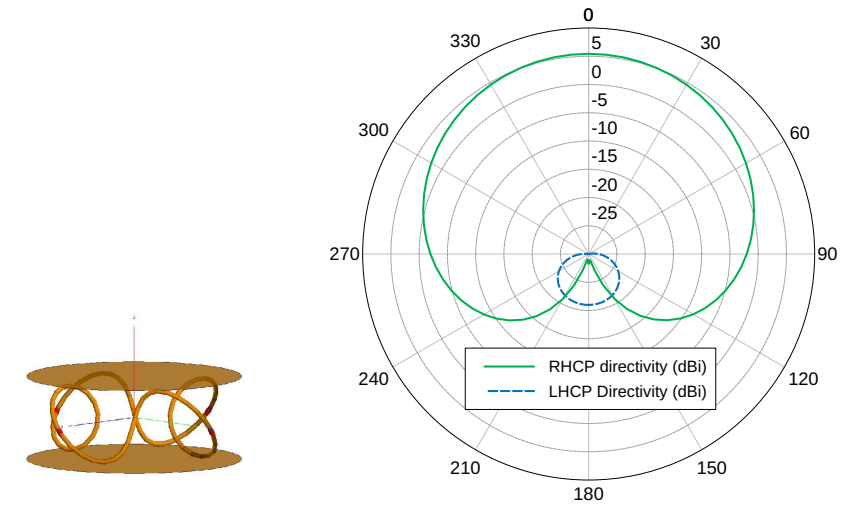

Fig. 7. Helical ring antenna with two extra metallic disks.

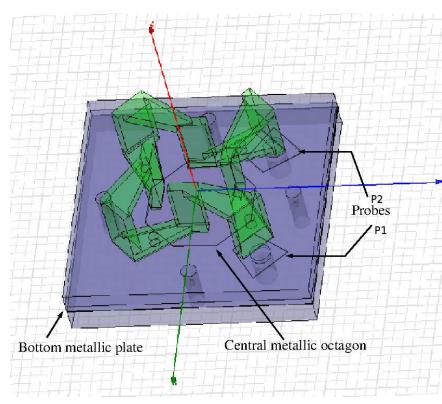

(a) 3D model

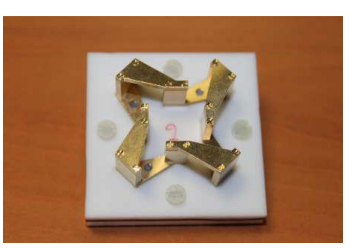

(b) picture
Fig. 8. Model and picture of the antenna.

- layer 2: a PTFE-glass board of thickness $1.524 \mathrm{~mm}$ and relative permittivity $\varepsilon_{\mathrm{r}}=2.55$, on which there are a metallic plate of octagonal shape, and two other plates $\left(P_{1}, P_{2}\right)$ associated with both feed probes;

- layer 3: a PTFE shim of thickness $3 \mathrm{~mm}$ and relative permittivity $\varepsilon_{\mathrm{r}}=2.1$;

- layer 4: the right-hand $\left(\alpha_{\mathrm{p}}=j\right)$ helical ring of outer diameter $38 \mathrm{~mm}$, inner diameter $5.5 \mathrm{~mm}$, height $13 \mathrm{~mm}$ and thickness $2 \mathrm{~mm}$.

The operating frequency is accurately adjusted by modifying the diameter of the central metallic octagon. Indeed, when the octagon diameter is increased, the helix becomes more capacitively loaded, and thus the resonance frequency is decreased.

In the previous section, the helical ring has ideally been excited by means of 4 voltage sources along the wire. In this section, a realistic excitation with only two probes is considered. In the prototype this is realized with two coaxial cables, which shields are connected to the aluminum support, and which cores are connected to either $P_{1}$ or $P_{2}$. In simulations, the two coaxial cables are modeled by two sources located between the aluminum supports and the plates $P_{1}$ and $P_{2}$. The size of these plates are adjusted so as to obtain an input impedance of $50 \Omega$. Alternatively, only one feed probe could be used by adding an 


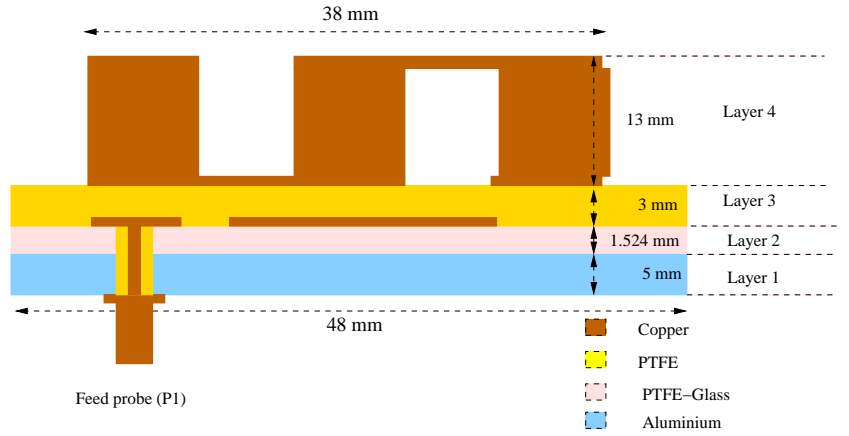

(a) side view

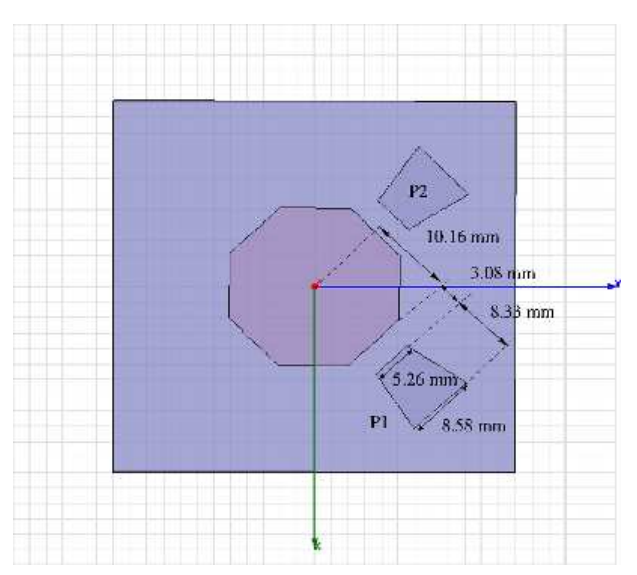

(b) top view of layer 2

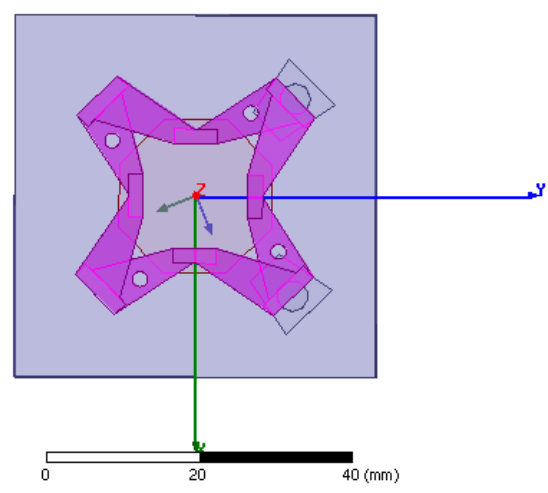

(c) top view of layer 4

Fig. 9. Side and top views of the antenna.

internal mode coupling to realize a quadrature excitation. The coupling element would then placed at $\pm 45^{\circ}$ from the position of the feed probe as presented in [13], with a \pm sign chosen to orient the radiation toward the direction $\pm O z$. A magnetic coupling would also be possible using classical small feeding loops. Capacitive coupling has been chosen here because it yields a simpler design.

Note that, dielectric layers are added for mechanical reasons. Their influence has been accounted in the choice of the antenna parameters to maintain the resonance frequency. Furthermore, simulation and measurements will confirm that the radiation principle is not significantly influenced by the dielectric layers.

In this section, the simulations are performed with HFSS and the measurements are realized in an anechoic chamber with a vector network analyzer (VNA).

\section{B. S-parameters}

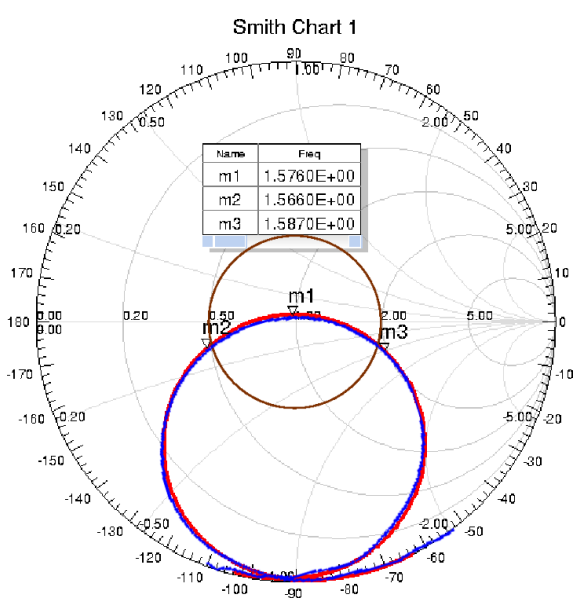

Fig. 10. Reflection coefficient $S_{11}$ on Port 1 of the antenna for a reference impedance of $50 \Omega$ : measured (blue), simulated (red). The brown circle indicates the area where VSWR $\leq 2$.

In Fig. 10, we display the measured and simulated values of $S_{11}$ determined from a reference impedance of $50 \Omega$. The value of the other $S$-parameters are so that $S_{22} \approx S_{11}$ and $S_{12}=$ $S_{21} \approx 0$ because of the symmetry of the antenna. The results obtained by means of simulations and measurements are in agreement. For both of them, the antenna central frequency is of $1.575 \mathrm{GHz}$ with a 2:1 VSWR bandwidth of $21 \mathrm{MHz}$.

\section{Radiation pattern}

All the simulated and measured radiation patterns of this section have been obtained at a frequency of $1.575 \mathrm{GHz}$.

1) Simulation results: In Fig. 11, the 3D gain pattern is plotted for a feeding such that $\alpha_{\mathrm{f}}=-j$. As expected, the antenna behaves as a Huygens source in circular polarization. The radiation is mainly RHCP and directed toward the $z \geq 0$ half-space with a cardioid pattern. The maximal gain is of $6.14 \mathrm{dBi}$. The difference of $1.37 \mathrm{~dB}$ with the theoretical value (10) may be explained by the presence of only one metallic support which size is larger than the outer diameter of the helical ring for mechanical purposes.

In Fig. 12, we display the radiation pattern for various azimuth angles and for $\alpha_{\mathrm{f}}=\mp j$. For both feedings, the radiation remains similar regardless of the azimuth angle. As previously, the direction of radiation can be electrically switched by modifying the sign of the in-quadrature feeding. Nevertheless, because of the presence of only one metallic support of large size, both feedings yield patterns that are not anymore symmetric to each other. The front-to-back ratio are of order $10 \mathrm{~dB}$ and $4 \mathrm{~dB}$ for $\alpha_{\mathrm{f}}=\mp j$, respectively. 


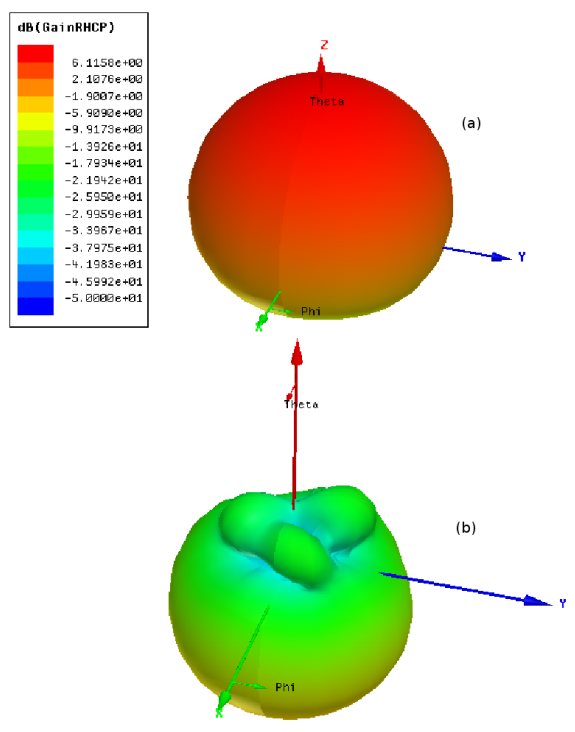

Fig. 11. 3D gain pattern (dBi) for $\alpha_{\mathrm{f}}=-j$ : (a) RHCP, (b) LHCP.

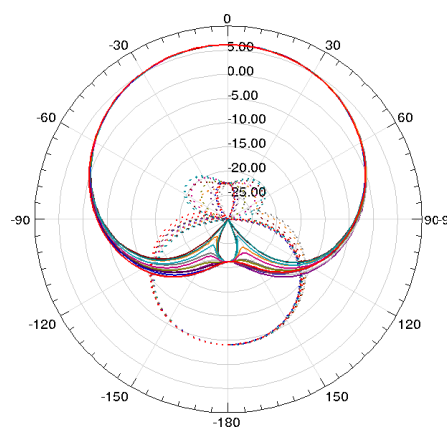

(a) $\alpha_{\mathrm{f}}=-j$

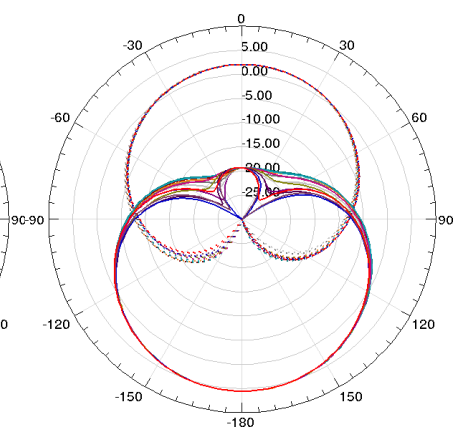

(b) $\alpha_{\mathrm{f}}=+j$
Fig. 12. Gain pattern (dBi) for azimuth angles $\phi=\left\{0,15^{\circ}, \cdots, 180^{\circ}\right\}$.

2) Comparison with measurements: The simulated radiation characteristics are now compared with measurements for which the in-quadrature feeding is obtained by means of a hybrid coupler. In Fig. 13, we compare the gain pattern in co and cross polarization in the upper half-space. The measurements are in very good agreement with the simulations. In simulation, the antenna efficiency is of $96 \%$. In measurements, removing the influence of the feeding circuit, we obtain an intrinsic efficiency of $93.4 \%$ for the antenna, which is consistent with simulations.

In Fig. 14, the measured and simulated axial ratio are plotted in the half-space $z \geq 0$. The radiation remains almost purely RHCP regardless of the direction in the the upper half-space even for angles near $\theta=+/-90^{\circ}$. The measured results are very similar and even better than the simulated ones. The $3 \mathrm{~dB}$ axial ratio beamwidth is larger than $180^{\circ}$.

\section{COMPACT HELICAL RING ANTENNA With 8 TURNS}

In this section, simulation results for a 8-turns helical-ring antenna are presented. The aim is to show that an increase in

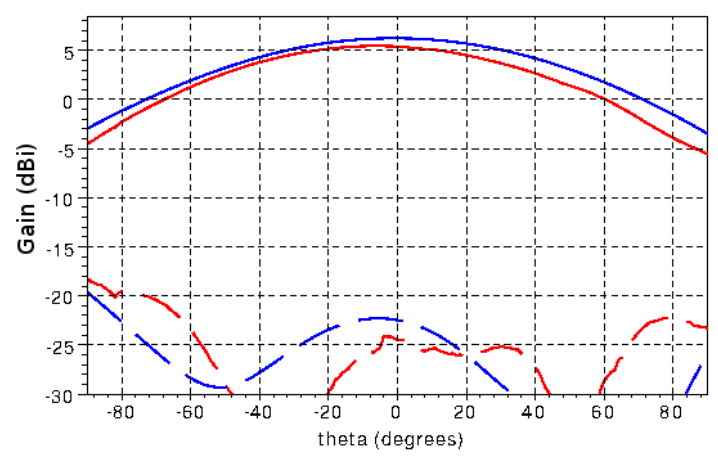

Fig. 13. Gain Pattern (dBi) in the $x O z$ plane for $\alpha_{\mathrm{f}}=-j$ : RHCP measurement (red line), RHCP simulation (blue line), LHCP measurement (dashed red line), LHCP simulation (dashed blue line).

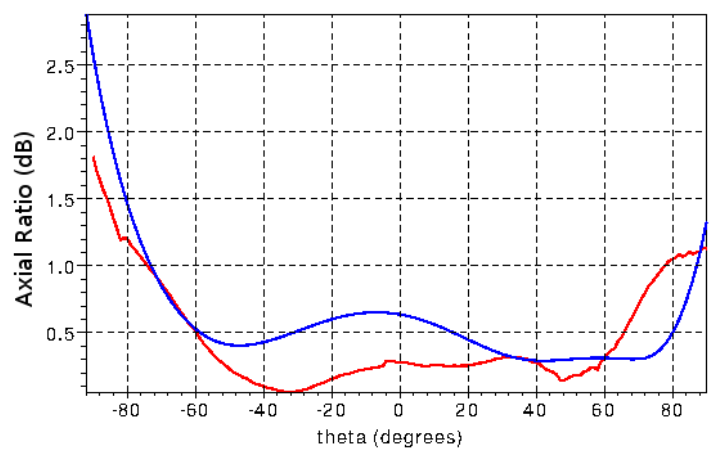

Fig. 14. Axial ratio (dB) in the $x O z$ plane for $\alpha_{\mathrm{f}}=-j$ : measurement (red), simulation (blue).

the number of turns reduces the size of the antenna without using high-dielectric materials that can be costly, heavy and lossy. This property has been shown for a straight helix in [14].

\section{A. Configuration}

As illustrated in Fig. 15, the antenna is symmetric and comprises two metallic disks. Two capacitive feed probes are placed between the helix and the bottom plate. Their size is adjusted so as to obtain an input impedance of $50 \Omega$ at resonance. The inner and outer radii of the helical ring are of $5.57 \mathrm{~mm}$ and $11.14 \mathrm{~mm}$, respectively. Besides, the helix height is of $5.57 \mathrm{~mm}$ and the gap between the disks and the helix is of $0.89 \mathrm{~mm}$. The helix is right-handed so that $\alpha_{\mathrm{p}}=j$.

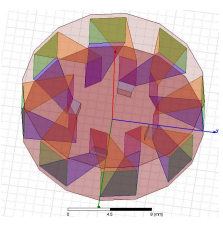

Fig. 15. Symmetrical antenna structure with eight turns. 


\section{B. Simulation results}

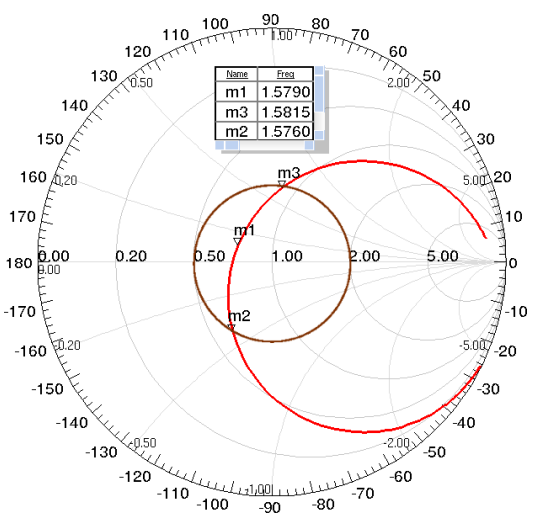

Fig. 16. Reflection coefficient $S_{11}$ on Port 1 of the antenna for a reference impedance of $50 \Omega$. The brown circle indicates the area where VSWR $\leq 2$.

In Fig. 16, The reflection coefficient on one of the ports is plotted in a Smith chart for a reference impedance of $50 \Omega$. The central frequency is of $1.579 \mathrm{GHz}$ and the $1: 2$ VSWR bandwidth is of $5.5 \mathrm{MHz}$. The size reduction is of $60 \%$ compared to the 4-turns antenna. Hence, an increase in the number of turns yields a smaller antenna at the cost of a reduced bandwidth.

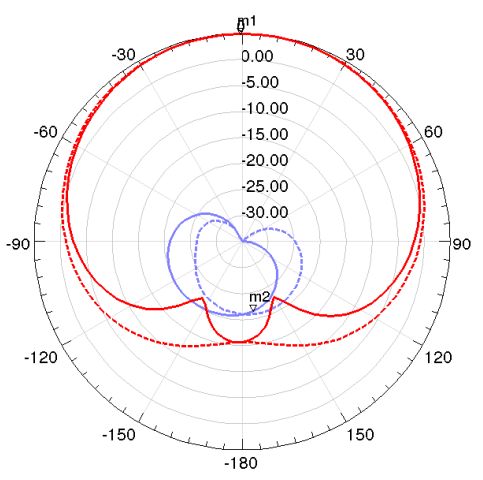

Fig. 17. Directivity pattern (dBi) for RHCP (red) and LHCP (blue) polarization, in the $x 0 z$ (solid line) and $y 0 z$ (dashed line) planes.

The directivity patterns are displayed for both circular polarizations in Fig. 17. The maximum directivity is about $4.95 \mathrm{dBi}$. The front-to-back ratio is of order $21.5 \mathrm{~dB}$. This value is better than the one obtained in the previous section. That may be explained by the presence of 2 metallic plates, which improves the antenna symmetry.

\section{CONCLUSION}

A helical ring antenna has been proposed to obtain a Huygens source in circular polarization. Its radiation pattern is mainly oriented toward one half-space without the use of any reflector. The direction of the radiation can be electrically switched frontward or backward by modifying the feeding. The principle of this antenna relies on two orthogonal modes, both of which radiate as collocated electric and magnetic short dipoles.

Analytic derivations have been developed to study this principle and to prove the cardioid pattern. They have been confirmed by means of simulations on a helical-ring wire. Simulations have also shown that two extra metallic plates can be added to simplify the balance between the electric and magnetic moments. Next, the design, simulation, realization and measurement of a 4-turns helical ring have been presented. The resonant frequency has been adjusted by modifying the coupling between the helical ring and a metallic plate. Both the measured and simulated characteristics match. For a total size of approximately $\lambda / 4$, the $3 \mathrm{~dB}$ axial-ratio-beamwidth is larger than $180^{\circ}$, the efficiency is about $94 \%$ and the relative bandwidth is of $1.33 \%$. Finally, simulations on a 8 turns helical ring have shown that increasing the number of turns reduces the size at the cost of a decreased bandwidth.

This antenna may be of interest when a very good circular polarization is required in an half-space, e.g. for satellitebased positioning systems (GNSS) or satellite phone communications (Iridium). Due to the capability of miniaturization without high-permittivity materials, this kind of radiating element could also be interesting for low-weight or low-frequency applications. Besides, the weak backward radiation and the small weight render this antenna adapted for body network applications.

\section{REFERENCES}

[1] P. R. Foster, and R. A. Burberry, "Antenna problems in RFID systems," in Proc. IEE Col. RFID Tech. (no. 1999/123.), London, UK, 1999.

[2] J. H. Bang, C. Bat-Ochir, H. S. Koh, E. J. Cha, and B. C. Ahn, "A Small and lightweight antenna for handheld RFID reader applications," IEEE Antennas Wireless Propag. Lett., vol. 11, pp. 1076-1079, 2012.

[3] K. C. L. Kwok-Chi Chim Chan, and R. D. Murch, "Investigating the impact of smart antennas on SAR," IEEE Trans. Antennas Propag., vol. 52, no. 5, pp. 1370-1374, May 2004.

[4] C. C. Kilgus, "Resonant quadrifilar helix," IEEE Trans. Antennas Propag., vol. 17, no. 3, pp. 349-351, May 1969.

[5] J. Rabemanantsoa, and S. Ala "Dual-band meandered folded printed quadrifilar helix antenna," in Proc. 5th Eur. Conf. Antennas Propag. (EUCAP), Rome, Italy, 2011, pp. 1828-1831.

[6] P. Alitalo, A. Karilainen, T. Niemi, C. R. Simovski, S. A. Tretyakov, and P. de Maagt, "Chiral antennas radiating circularly polarized waves," in Proc. 4th Eur. Conf. Antennas Propag. (EUCAP), Barcelona, Spain, 2010, pp. 1-5.

[7] P. Alitalo, A. O. Karilainen, T. Niemi, C. R. Simovski, and S. A. Tretyakov, "Design and realisation of an electrically small Huygens source for circular polarisation", IET Microwave Antennas Propag., vol. 5, issue 7, pp. 783-789, May 2011.

[8] B. Souny, C. Morlaas, and A. Chabory, "Compact self-directional antenna based on a helical ring," in Proc. Antennas and Propagation Soc. Int. Symp. (APS/URSI), Toronto, Ontario, Canada, 2010, pp. 1-4.

[9] B. Souny, "Antenne autodirectrice en polarization circulaire - Self-directing antenna with circular polarization," Patent FR0904076, EP2293385, Aug. 2010 [online]. Available: http://www.google.com/patents/EP2293385A1?cl=fr

[10] H. A. Wheeler, "A helical antenna for circular polarization," in Proc. IRE, vol. 35, no. 12, pp. 1484-1488, Dec. 1947.

[11] J. D. Kraus, "The helical antenna," in Proc. IRE, vol. 37, no. 3, pp. 263-272, March 1949.

[12] J. E. Storer, "Impedance of thin-wire loop antennas," American Institute of Electrical Engineers Trans., vol. 75, no. 5, pp. 606-619, Nov. 1956.

[13] C. Morlaas, A. Chabory, and G. Hattenberger, "Compact helical ring antenna for iridium communication on UAV," in Proc. European Test and Telemetry Conf. (ETTC), Toulouse, France, 2011.

[14] M. Pigeon, C. Morlaas, H. Aubert, and B. Souny, "Highly compact composite antenna," in Proc. Antennas and Propagation Soc. Int. Symp. (APS/URSI), Charleston, SC, USA, 2009, pp. 1-4. 


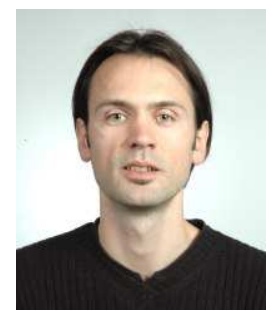

Christophe Morlaas received the Ph.D. degree in electronics from ISAE-SUPAERO ("InstitutSupérieur de l'Aéronautique et de l'Espace") in 2000. He hold a system engineer position in spatial programs until 2002. Since then, he is an assistant professor with the Electromagnetics and Antennas Research Group of the Telecom lab of ENAC. His research interests include aeronautical communication and navigation systems, electromagnetic modeling, propagation models and antenna design.

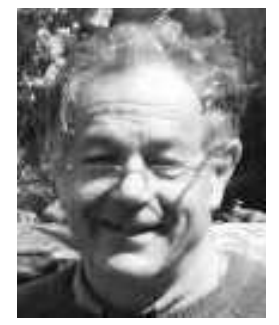

Bernard Souny graduated in 1973 as an electronics engineer from the French Civil Aviation University (ENAC). In 1993, he received the Ph.D degree from INPT (Institut National Polytechnique de Toulouse). He headed the LETA laboratory of ENAC. Since 2010, he is retired and remains an associate researcher with ENAC. His research interests include computational electromagnetics, antenna design and propagation models.

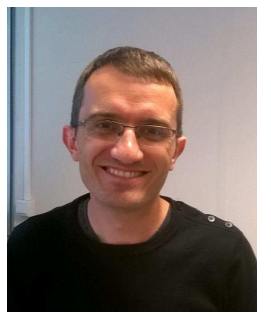

Alexandre Chabory graduated in 2001 as an electronics engineer from the French Civil Aviation University (ENAC). From 2001 to 2004, he was a PhD student with ONERA. From 2004 to 2007, he was a postdoctoral scientist with the Eindhoven University of Technology (TU/e). Since 2007, he is an assistant professor with the Electromagnetics and Antennas Research Group of the Telecom lab of ENAC. Since 2012, he heads this group. His research interests deal with electromagnetic theory and modeling, mainly for aeronautical applications. 